\title{
Posttranscriptional Control of Gene Expression and Role of Small RNAs in Streptococcus mutans
}

\author{
Jarosław E. Król \\ Center for Advanced Microbial Processing, Department of Microbiology and Immunology, Drexel University College \\ of Medicine, Philadelphia, PA, USA \\ Email: Jek322@drexel.edu
}

How to cite this paper: Król, J.E. (2018) Posttranscriptional Control of Gene Expression and Role of Small RNAs in Streptococcus mutans. Advances in Microbiology, 8, 138-160.

https://doi.org/10.4236/aim.2018.82010

Received: December 29, 2017

Accepted: February 25, 2018

Published: February 28, 2018

Copyright $\odot 2018$ by author and Scientific Research Publishing Inc. This work is licensed under the Creative Commons Attribution International License (CC BY 4.0).

http://creativecommons.org/licenses/by/4.0/

\section{cC) (i) Open Access}

\begin{abstract}
Streptococcus mutans is a key contributor to the formation of biofilms associated with dental caries disease. Living in the oral environment and developing of disease require tight gene regulation. In bacteria, gene expression is most commonly regulated at the level of transcription initiation. This control involved interactions of specific DNA sequences with regulatory proteins. A second mechanism of control of gene expression is mediated at the RNA level by several mechanisms and is generally called posttranscriptional regulation. These mechanisms include cis- and trans-acting small, non-coding RNAs, RNA-binding proteins, riboswitches, thermosensors, RNases, and Type I toxin-antitoxin systems, and may result in changes in RNA stability, efficiency of ribosome binding, translation initiation, and transcript secondary structures. Here I review the posttranscriptional regulation in $S$. mutans.
\end{abstract}

\section{Keywords}

Dental Carries, Gene Regulation, Antisense Therapy

\section{Introduction}

The general rules of life are the same for all living organisms. Everything that is true for bacteria, is true for big animals as well. That theory coined in the XIX century resulted in focusing scientific research on model organisms which were easy to cultivate (propagate), stable and amenable to experimental manipulation. Our knowledge on bacterial physiology is based mostly on two models: Escherichia coli, a Gram-negative rod, which is a common constituent of the human digestive system and Bacillus subtilis, a Gram-positive, rod shaped, sporulating, 
free living organism. Due to an enormous progress in DNA sequencing technologies the last decade allowed researchers to establish and annotate hundreds of microbial genomes. Based on that data it can be noticed that many genes are specific for a specific organism, have no similarities with other genes and no function can be assigned. That leads to the conclusion that many organisms are unique and do not undergo the common paradigms. One of those organisms, Streptococcus mutans, has been proposed to be a new model organism for Gram-positive pathogenic bacteria [1].

It has been almost 90 years since $S$. mutans was described for the first time in 1924 and almost thirty years since its role in formation of dental caries was established [1]. The last decade, which started with the first completed genome sequence [2], revealed many interesting features of the $S$. mutans specific physiology. Developing many new molecular tools and usage of the "tip top" molecular biology techniques also contribute to understanding of those processes on molecular level [1] [3]-[10]. That knowledge leads also to developing new therapies for human carries [11] [12] [13] [14].

Regulation of gene expression is fundamental for the coordinate synthesis, assembly and localization of all macromolecular structures of cells. This is achieved by a multi-step program that is highly interconnected and regulated at diverse levels. In bacteria, gene expression is most commonly regulated at the level of transcription initiation. This control involved interactions of specific DNA sequences with regulatory proteins. A second mechanism of control of gene expression is mediated at the RNA level by several mechanisms and is generally called posttranscriptional regulation. These mechanisms include cis- and trans-acting small, non-coding RNAs, RNA-binding proteins, riboswitches, thermosensors, RNases, and Type I toxin-antitoxin systems, and may result in changes in RNA stability, efficiency of ribosome binding, translation initiation, and transcript secondary structures [15] [16] [17] [18] [19].

In $S$. mutans UA159 genome more than 100 different transcriptional regulators have not has been identified [2]. Only two RNA polymerase sigma factors have been found: $\sigma^{70}$ (RpoD: SMU.822 and SMU.1803) and ComX (SMU.1997), a transcriptional regulator of competence-specific genes [2]. The major role in gene regulation and adaptation to environmental conditions in $S$. mutans is being held by the RpoE, delta subunit of RNA polymerase [20] [21] and two component signal transduction systems (TCSTS). The role of each of the 14 TCSTS as well as a single orphan regulator GcrR has been studied extensively and recently reviewed [22]. Here I am going to shed some light on a question of posttranscriptional regulation of gene expression in $S$. mutans.

\section{Mechanisms of Posttranscriptional Regulation}

For many bacterial operons, the mechanism of posttranscriptional control of gene expression relies on sequence signals in the 5 '-untranslated region (5'-UTR) found upstream of gene coding sequences. These leader regions can fold into at 
least two mutually exclusive RNA structures, like an intrinsic terminator and an anti-terminator. Switching between different conformations of this transcribed region controls the fate of the elongation complex, and thus the expression of the downstream gene. This switch is controlled by diverse regulators that include accessory proteins, small molecules, uncharged tRNAs and translating ribosomes [23] [24] [25]. Another mechanism of posttranscriptional regulation involves regulatory proteins which bind to mRNA and block its translation initiation region (negative regulation) or stabilize mRNA molecule and/or structure (positive regulation) [26]. The third mechanism involves the complementary RNA molecules (asRNA, sRNA, miRNA) that action similar to RNA binding proteins but may also target a complementary mRNA strand for degradation (RNA decay) [27] [28] [29].

\section{Regulation by RNA Binding Proteins}

\subsection{BglG(LicT)/SacY Antiterminator Proteins}

Antiterminator (AT) proteins modify the structure of the target mRNA in order to prevent formation of the termination signals and subsequent dissociation of the polymerase. However direct interaction with the transcription complex is not required in that case. The best known bacterial AT belongs to the $\mathrm{BglG}(\mathrm{LicT}) / \mathrm{SacY}$ family, which has been shown to be involved in carbohydrate transport and metabolism in many Gram-positive and Gram-negative bacteria [30]. The RNA target sequences (called RAT-ribonucleic antiterminator) for these proteins are usually 30 nucleotides long and are proposed to adopt a hairpin structure with a variable apical loop and two asymmetric internal loops interrupting a central stem [31]. Mutational analysis on different RATs has shown that base pairings in the stem are required for the antitermination function and that the non-conserved nucleotides within the internal loops are involved in the control of the specificity of the AT-RAT interaction. In contrast, RAT recognition by the AT appears largely independent of the length and nucleotide sequence of the apical loop [32].

In the genome sequence of $S$. mutans UA159 five genes encoding AT proteins and fourteen PTS systems have been identified [2] [32]. Two of those PTS are responsible for utilizing $\beta$-glucosides such as cellobiose, esculin, arbutin, and salicin which can be found in foods containing plant extracts. Cellobiose and salicin are utilized by proteins encoded in the cel locus (SMU.1596 to -1601). The second locus, designated bgl (SMU.977 to -985), consists of the genes for PTS transport (EII, SMU.980) and the metabolism of esculin. Arbutin is hydrolyzed by its own phospho- $\beta$-glucosidase (encoded by the arb gene, SMU.1102), but it is transported by the cel or $b g l$ system [33]. Two out of those five identified AT genes are located within the cel and $b g l$ operons (SMU1599-celR and SMU977) and one is located within $m t l$ operon (mannitol operon $m t I R$-SMU1184) while the fourth is encoded by a single SMU289 gene located $\sim 8 \mathrm{~kb}$ downstream of the putative ribulose utilization operon (SMU270-275). The mechanism of action between 
AT and the RAT sequence have been described only for the SMU977-LicT in $S$. mutans NG8 [34] [35] [36]. Based on DNA sequence homology between NG8 and UA159 strains it was possible to identify the $b g l$ RAT structure and the terminator in front of the $b g I P$ gene (Figure 1(a)). Another hypothetical RAT sequence has been described in front of a fructan hydrolase gene (fruA) [37]. However further experiments showed that this structure was not involved in gene regulation [38].

The role of CelR in regulation of the cel operon has been shown recently [39]. Although the detailed mechanism of interaction has not been determined a similarity with the MtlR protein of Bacillus stearothermophilus (29\% identity) suggests that CelR might act as the transcriptional regulator at the DNA level [39] [40]. The latest data on Vibrio parahemeolyticus MtlR protein structure and function showed that it does not bind to the promoter regions directly [41]. Therefore, identification of the hypothetical RAT and terminator structures in the celA mRNA leader (Figure 1(b)) might suggest the antitermination mechanism of regulation.

The mechanism of action of three remaining AT proteins, MtlR, SMU289 and SMU787, which show limited homologies to other proteins is not known. Although the location of $S$. mutans MtlR protein suggests its role in regulation of mannitol operon, it shows even lower similarity level with MtlR B. stearothermophilus (23\% identity) than CelB. A target sequence for the orphan regulators, SMU787 and SMU289, remains unknown but a presence of the fluoride riboswitch in front of SMU787 might suggest its role in fluoride resistance (see riboswitch section).

\subsection{Ribosomal RNA Regulation-NusAB Antitermination}

Transcription termination factors NusAB (N protein-Utilization Substance AB) have been described for the first time in bacteriophage lambda [42]. Further studies showed that the same system plays a key role in the regulation of ribosomal RNA biosynthesis in eubacteria by modulating the efficiency of transcriptional antitermination [43]. NusB along with other Nus factors (NusA, NusE/S10 and NusG) forms the core complex with the boxA element of the nut site of the rRNA operons [44] [45]. These interactions help RNA polymerase to counteract polarity during transcription of rRNA operons and allow stable antitermination. Although the Nus proteins recognize the boxA sequence in rRNA leader they act rather as transcription elongation factors modifying RNA polymerase sensitivity to the presence of rho-dependent transcription termination sites [46].

Five rRNA operons have been identified in the $S$. mutans UA159 genome [2]. Also all three components of the antitermination system: NusA (SMU.418), NusB (SMU.1845) and NusG (SMU.1947) have been described [2]. Although blast search revealed many sequences identical with the original boxA sequence $\left(5^{\prime}-(\mathrm{t} / \mathrm{c})\right.$ GCtCTTtaaca(a/t)(T/C)-3'), none of them was actually located in the vicinity of the rRNA genes. Therefore, the role of Nus antiterminators, as well as rRNA transcription regulation in $S$. mutans, remains unknown. 


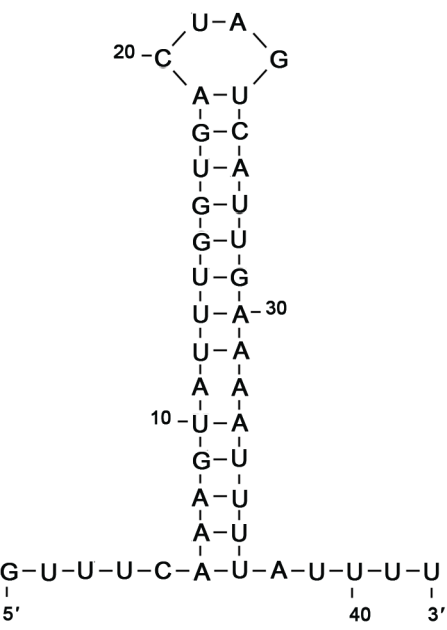

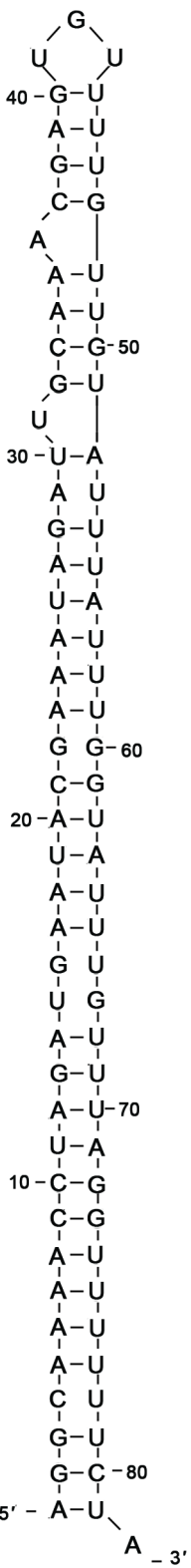

(b)$$
\text { ) }
$$

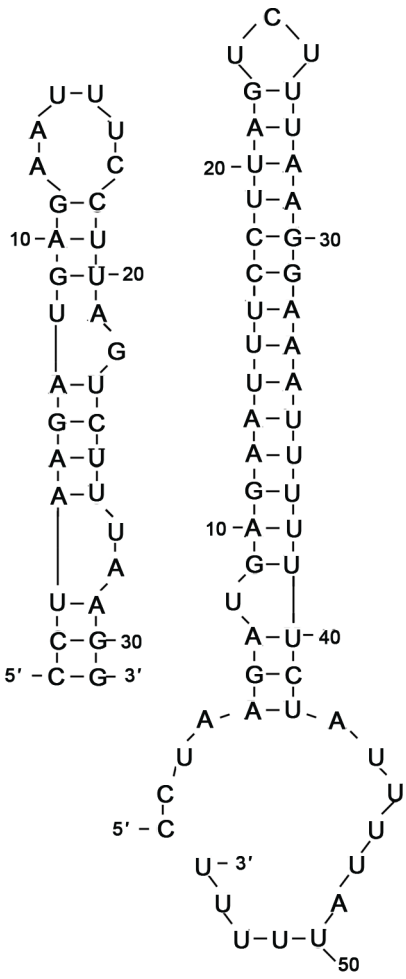

(a)

(c)

Figure 1. RAT structure and terminator of $S$. mutans UA159 bgIP mRNA (a) and a hypothetical RAT structure and terminator in the celA leader (b).

\subsection{Termination Protein-PyrR Regulon}

The PyrR protein regulates expression of the genes of de novo pyrimidine nucleotide biosynthesis ( $p y r$ genes) in almost all Gram positive and many other bacteria by a transcription attenuation mechanism [47]. PyrR acts by binding to a segment of pyr mRNA with conserved sequence and secondary structure. 5 '-UTR leader folds to form three alternative structures, an antiterminator, a terminator, and an anti-antiterminator that precedes the antiterminator [48]. The PyrR protein, in the presence of UMP, can bind to, and stabilize, the anti-antiterminator. This prevents formation of the antiterminator, resulting in 


\subsection{Metabolite-Sensing Riboswitches}

In many bacteria genes involved in vitamins production and transport, as well as amino acid and nucleotides biosynthesis are regulated by metabolite-sensing riboswitches. Specific sequences responsible for binding flavin mononucleotide (FMN, rfn-box), thiamin pyrophosphate (TPP, tpp-box), adenosylcobalamin (B12, B12-box), $S$-adenosyl-1-methionine (SAM, S-box; $\mathrm{S}_{\mathrm{MK}}$-box), lysine (L-box), guanine and hypoxanthine (Purine, G-box) have been identified [54] [55] [56].

In $S$. mutans UA159 genome sequence originally four metabolite-sensing riboswitches, FMN, TPP, Glycine and yybP/ykoY have been identified (http://www.oralgen.org/srna_result/index.html\#S.mut). The TPP riboswitch has been found upstream of SMU706 gene. It shows similarity to yuaJ B. subtilis encoding for thiamine permease [57]. FNM riboswitch is located in front of the SMU1703-1702 operon (1615112-1615325). SMU1703 shows homology to ypaA gene from $B$. subtilis which is a membrane protein involved in riboflavin uptake [58]. Glycine riboswitch typically resides upstream of genes that express protein components of the glycine cleavage system which catalyzes the initial reactions for use of glycine as an energy source like B. subtilis $g_{c v} T-g_{c v} P A-g_{c v} P B$ operon. However in Vibrio cholerae it is located immediately upstream of the VC1422 gene (a putative sodium and alanine symporter) [59]. In $S$. mutans, Glycine riboswitch is located in front of the SMU1175 gene which is similar to VC1422. The last riboswitch element $y y b P / y k o Y$ is common and widely distributed in other bacteria. The role of that riboswitch and the mechanism of regulation have not been described. The yybP/ykoY element resides upstream of two separate monocistronic transcripts in B. subtilis and E. coli and is found mainly upstream of genes classified into four groups. The first cluster includes $E$. coli ygjT and $B$. subtilis $y k o Y$, which are similar to an $E$. coli gene (terC) that encodes a membrane protein with a poorly defined function related to tellurium resistance. The second group encodes a cation-transport ATPase, whereas the final two clusters are predicted to be membrane proteins (one includes E. coli yebN) [60]. In $S$. mutans yybP/ ykoY element is located upstream of SMU.723 gene encoding for a putative calcium-transporting ATPase [2]. Location of that riboswitch in front genes involved in cation transport might suggest the role of cations in its activation. Other riboswitches involved in sensing intracellular level of ions like magnesium riboswitch described in Salmonella enterica [61] have not been so far identified in $S$. mutans.

Additional studies revealed the presence of additional three riboswitches in $S$. mutans. Although a standard S-box has not been identified in $S$. mutans genome, Fuchs and coworkers found an alternative $\mathrm{S}_{\mathrm{MK}}$-box in front of metK gene (SMU1573) encoding for S-adenosylmethionine synthetase [62]. Recently, an additional riboswitch interacting with tetrahydrofoliate (THF) that controls the folT gene in Streptococcus mutans UA159 have been identified [63] [64]. Two molecules of folinic acid, THF analog, were observed to bind to that structure and prevent synthesis of genes involved in THF transport and synthesis [63]. 
Another newly described riboswitch, $\operatorname{crcB} / \operatorname{eri}^{F}$ senses fluoride concentrations. The $c r c B$ motifs are located upstream of genes encoding proteins annotated as ion transporters (for example, chloride, sodium, proton). Some others are involved in various physiological (e.g., universal stress adaptation, DNA repair) or metabolic (e.g., enolase, formate-hydrogen lyase) processes [65]. In Streptococcus mutans UA159 the eriC riboswitch has been shown to regulate SMU.787 gene [65]. The SMU.787 protein is a transcriptional regulator related to cell envelope and contains transcriptional attenuator domain [2]. As fluoride has been widely used as an additive in oral hygiene products and water since the 1950s because of its usefulness in preventing tooth decay [66], it would be important to reveal the role of that riboswitch in $S$. mutans fluoride toxicity resistance.

\subsection{T-Box Regulation Mechanism}

The T-box mechanism is widely used in Gram-positive bacteria to regulate expression of aminoacyl-tRNA synthetase genes and genes involved in amino acid biosynthesis and uptake. Binding of a specific uncharged tRNA to a riboswitch element in the nascent transcript causes a structural change in the transcript that promotes expression of the downstream coding sequence. In most cases, this occurs by stabilization of an antiterminator element that competes with formation of a terminator helix. Specific tRNA recognition by the nascent transcript results in increased expression of genes important for tRNA aminoacylation in response to decreased pools of charged tRNA [67] [68].

In $S$. mutans UA159 genome sequence originally four T-boxes in front of the trp operon as well as Ala-, Thr- and Ser-tRNA synthetases have been identified (http://www.oralgen.org/srna_result/index.html\#S.mut). In other studies ten T-box sequences ( 8 for AA-tRNA synthetases, 1- AA synthesis and 1- unknown function) were described [69]. In total 10 AA-tRNA synthetases are regulated by the T-box mechanism (AlaS, AsnS, GlyS, HisS, AspS, IleS, PheS, SerS, ThrS and $\mathrm{ValS}$ ). Some of those genes constitute polycistronic transcriptional units.

T-box regulates also histidine biosynthesis operon. That mechanism is specific for Lactobacillales, including L. lactis and $S$. mutans [69]. The his operon in $S$. mutans contains also genes for serine biosynthesis (serB) and hisC and his $H$, which encode the enzymes that catalyze some of the last steps of the tyrosine, and phenylalanine pathways. The rarity of T-box regulation for his operon may be caused by the fact that the acceptor arm of tRNA ${ }^{\text {His }}$ has only three unpaired nucleotides (CCA) at its 3 ' end. Therefore, $\mathrm{tRNA}^{\text {His }}$ lacks one of the unpaired nucleotides involved in the interaction of tRNAs with the antiterminator bulge. The absence of the fourth position of pairing could potentially result in a lower efficiency of tRNA-dependent antitermination [69].

The T-box sequence identified upstream of the trpEGDCFBA operon (SMU532-538) was located upstream of the small gene SMU531 with similarity to chorismate mutase $(p h e A)$. As that gene location is unique only to Streptococcus thermophilus and $S$. mutans it has been speculated that SMU531 had 
been horizontally transferred and inserted in front of $\operatorname{trpE}$ gene [69].

\section{Antisense RNA}

Antisense RNA (asRNA) is a single-stranded RNA that is complementary to a messenger RNA (mRNA) strand. Antisense RNA reflects usually to long transcripts in contrast to small interference RNA (RNAi); however the mechanism of action is probably similar [70] [71]. The simplest example of such antisense structure is when two adjacent genes overlap and they are transcribed in the opposite direction. There are two possible conformations for those overlapping genes: "convergent" $(\rightarrow \leftarrow)$ and "divergent" $(\leftrightarrow \rightarrow)$. Analysis of 50 microbial genomes showed that while co-transcribed, directly overlapping genes represent $71.4 \%$, the two inverted orientations have the same numbers (14.3\%) of gene pairs, but the fraction of the divergent overlapping genes $(\leftarrow \rightarrow)$ is lower than that of the convergent overlapping genes $(\rightarrow \leftarrow)$ [72]. The lower ratio of the divergent structure is probably due to the evolutionary constraints on the 5 'end of the gene and the upstream region, which have structures that are essential for the gene expression [72]. Although these data represents only annotated ORFs which contain translation signals (RBS and start codon) while those signals are not required in asRNA, the number of divergent transcripts should not dramatically increase. However, like other regulatory genes, expression of many asRNAs depends on cell physiology and environmental conditions, advances of new microarray and whole transcriptome sequencing techniques should allow the precise identification of those molecules.

\section{Toxin-Antitoxin System}

The role of toxin-antitoxin systems in cell physiology, specifically in biofilm formation, persister cell formation and the general stress response, is becoming clearer [73] [74]. So far, five types of toxin-antitoxin (TA) systems have been described [75] [76] [77] [78] [79]. In two TA types (I, III) the RNA molecules play important roles.

In type III a 36 nt ToxI antitoxin RNA repeat sequesters ToxN protein antitoxin and prevent its cytostatic activity [80]. In type I TA systems, antitoxins are small untranslated RNAs acting as asRNAs. RNA antitoxins can be encoded directly opposite to the coding sequence of the toxin, opposite the 5 -UTR, or opposite the 3 -UTR of the toxin mRNA, or even divergent to the toxin gene but with long stretches of complementarity to the toxin mRNA [81]. First described systems were involved in stability of plasmids in Enterobactericeae [81].

Although in $S$. mutans plasmid encoded TA system belongs to the type II, RelBE $_{\text {plas }}$ family [82], the presence of type I TA system has been recently reported [83]. The system named Fst-Sm/srSm is encoded by a small intergenic region (318 nt) located between the genes SMU.219 and SMU.220. Although the regions of base pairing between $s r S m$ RNA and the 5 ' end of $f s t-S m$ mRNA suppresses toxin translation, the mechanism of action has not been determined [83]. 
Both mechanisms of action, translational silencing or mRNA degradation as well as their combination are probable. The srSm:fst-Sm duplex is a good target for cleavage by RNase III, an endoribonuclease specific for double stranded RNA which is encoded by SMU.1415 gene. Attempts to construct a $S$. mutans RNase III mutant were unsuccessful, suggesting that this gene may be essential in $S$. mutans [83].

\section{Small Non-Coding RNA}

The bacterial non-coding RNA transcripts are usually 80 - $100 \mathrm{nt}$ in length and are not generally processed. Many sRNAs like $\operatorname{oxy} R, \operatorname{ryh} B, \operatorname{dsr} A, \operatorname{sgr} S$, RNAIII, pel or fas $X$ have key roles in the bacterial response to stress and regulation of factors important for virulence [84] [85] [86]. Many of those RNAs interact with small protein Hfq protein which increases their activity [87] [88]. So far neither $h f q$ gene nor any of the well known small RNAs has been described in $S$. mutans, even if they are present in other Streptococci [89] [90] [91] [92]. Lemme and co-workers showed that the expression of 30 intergenic regions was significantly changed in a cell fraction where expression of $\operatorname{com} X$ gene was induced by CSP protein [93]. When the transcriptome of cells induced by CSP was compared to that of non-induced cells much more (380) differentially expressed intergenic regions were found [93]. Although no detailed analysis has been conducted that data suggests a potential role of small non-coding sequences in gene regulation of CSP induced pathways.

\subsection{Clustered, Regularly Interspaced Short Palindromic Repeats (CRISPRs) Immunity—crRNA and TracrRNA}

CRISPRs motifs, discovered in late 1980s, are involved in bacterial immunity mechanisms to protect cells against invasion of an "alien" DNA. That RNA -based adaptive defense system which targets phages or plasmids has been identified in almost $40 \%$ of currently sequenced bacterial genomes [94]. Currently eight types of CRISPR have been described [95]. The major part of the system contains a different number of $31 \mathrm{bp}$ (23 to 50) long CRISPR motifs separated by an average 36 bp (17 to 84) long spacer sequences [96] The spacers show similarities to phage and plasmid sequences that can infect a particular cell. Typically, a repeat cluster is preceded by a long "leader" sequence. The second part constitutes genes encoding CRISPR associated proteins (cas genes) which are involved in both immunity acquisition and function. The mechanism of immunity acquisition is not known. It has been shown that new units (one or a few) are attached to the proximal (5') region and some Cas proteins are necessary in that process [94]. After activation the CRISPR region is transcribed as pre-crRNA and undergoes maturation. The final form of crRNA molecule contains an 8-nt part of the CRISPR motif on its 5' end, a spacer region and another part of CRISPR on the 3 ' end. For some types of CRISPR, Cas proteins alone are responsible for the maturation. Recently it has been described that maturation of 
csRNAs in $S$. pyogenes type II CRISPR (CRISPR01) depends on the presence of a small -171- and 89- nt long tracrRNA as well as the RNase III and Crn1-Cas protein [97]. After maturation csRNP (csRNA/Cas) complexes are able to recognize and inactivate any foreign DNA (RNA) with homology to the spacer region however the molecular mechanism of that process still remains elusive.

The annotated genome of $S$. mutans UA159 was found to harbour two CRISPR loci, designated CRISPR1 and CRISPR2 [2] [98]. CRISPR1 is located between ORFs SMU.1400 and SMU.1398, and contains seven copies of a partially palindromic sequence of $36 \mathrm{bp}$. All repeats are interspersed by spacers of 30 bp in size [98]. Four cas genes have been identified upstream of CRISPR1 (SMU.1405-csn1, SMU.1404-cas1, SMU.1403-cas2 and SMU.1402-csn2). S. mutans CRISPR1 shows similarity with $S$. pyogenes CRISPR01 and the tracrRNA gene has been identified upstream of the SMU.1405 gene [97] [98]. The fact that, in contrast to $S$. mutans, the RNase III mutant of $S$. pyogenes has been described might suggest a different role of that enzyme in these two species [83] [97].

The CRISPR2 locus of UA159, a member of the type 3 CRISPR, is located between SMU.1753c and SMU.1752c, and consists of two 32 bp partially homologous repeats separated by a 34 bp spacer [98]. The expression of cas genes of the CRISPR2 was upregulated in a strain defective in $c l p P$ protease [99].

\subsection{Cia-Dependent Small RNA (csRNAs)}

These small RNAs were originally described in Streptococcus pneumoniae. The first csRNAs were found in the regulon of the two-component regulatory system (TCS-TCSTS) CiaRH [100] and were designated csRNAs (cia-dependent small RNA). They show a high degree of similarity to each other, especially in the unpaired region between the two stem-loop structures characteristic for these sRNAs. Complementarity to the translation initiation region (TIR)-Shine-Dalgarno (SD) sequence and the start codon AUG within this unpaired region suggested that the csRNA could control translation initiation of mRNAs.

In Streptococcus mutans, the CiaRH system is involved in bacteriocin production, competence regulation, biofilm formation, and tolerance to environmental stresses [22]. The search for csRNAs in $S$. mutans genome sequence revealed the presence of 3 csRNA molecules-csRNA23-1 (79nt), csRNA23-2 (81nt) and csRNA24 (144nt). CsRNA23-1 and csRNA23-2 shows a high degree of similarity on both sequence and structure levels. They form 2 stem-loop structures on both 5' and 3' ends. CsRNA24 is much longer and contains 4 stem-loop structures. Despite the size and structural differences the sequence complement to the TIR shows high degree of similarity. Therefore potential target prediction with the sTarPicker software (https://omictools.com/starpicker-tool) revealed 162 hypothetical target genes $(\mathrm{P}>0.5)$ that were identical to CsRNA23-1, csRNA23-2 and csRNA24. It is not surprising that genes involved in processes regulated by the CiaRH system like bacA1 and SMU.1341 involved in bacteriocin production, $\operatorname{com} B$, com $Y B$-competence, $g b p A$-biofilm formation and $g r p E$-stress re- 
sponse were identified among these targets. The presence of genes belonging to some sugar PTS systems (like SMU.102, SMU.872, SMU.1598, SMU.1600, SMU1491, SMU.977) and specific regulators like MalR, LacR, GalR might suggest that csRNAs are involved in sugar metabolism. Eight more regulatory genes with $r p o D$ gene encoding for housekeeping, sigmaA RNA polymerase subunit identified among csRNA targets also suggest that the role of those small RNAs is more complex.

It is worth to noting that some of the target sequences are localized within long transcriptional units. Therefore, one of the possible functions of these sRNA might be differentiation of protein translation levels within a single operon. In a single polycistronic mRNA the difference in number of proteins synthesized from the consecutive genes relies on the affinity of ribosome to the TIR regions. The presence of csRNA molecule in the TIR region might modulate that interaction and subsequently change the protein amount produced by that specific gene. It is also interesting that in several different TCSTS sRNA have been described to modulate transcription of activated genes [101] therefore that mechanism seems to be more common.

\section{MicroRNAs}

MicroRNAs (miRNAs) are small noncoding RNAs with a size of $22 \mathrm{nt}$ found in many living organisms and function as an important modulators of gene expression mostly in Eukaryota [102] [103] [104]. Advantages of high-throughput transcriptome sequencing methods (RNAseq) allow identifying miRNAs and analyzing their role in bacterial physiology. Recently, Lee and Hong have analyzed small transcripts in $S$. mutans and they identified 922 miRNAs [105]. Despite the possibility that some of those molecules resulted from the processing of long mRNA transcripts, the presence of some of those miRNA species in a great number might suggest that they are not simply a degradation products. As the role of miRNAs in eukaryotic cells has been established it is important to study those systems in bacterial cells to reveal their functions and possible phylogenetic relations.

\section{Housekeeping Small RNAs}

Some sRNAs play an important role in regulation of important physiological processes and they are widely distributed among different taxa therefore they were named housekeeping sRNAs. The most known housekeeping RNA are ribosomal RNAs, RNaseP, tmRNA, 6S RNA and SRP RNA.

\section{1. tRNA Processing-RNase $P$}

A proper structure of tRNA molecules necessary for its transfer function and T-box regulation is maintained by two RNases: exoribonuclease-RNase $\mathrm{P}$ and endoribonuclease-RNase $\mathrm{Z}$. RNase $\mathrm{P}$ is a ribozyme and contains two components: an RNA chain, called M1 RNA, and a polypeptide chain, or protein, called 
C5 protein [106]. Both components have been identified in S. mutans genome (M1 RNA pos. 439800-440179; C5, rnaP-SMU336) [2]. The major activity of RNase $\mathrm{P}$ is processing of the 5 'leader sequence of precursor tRNA. The 3' end of tRNA, on the other hand, is processed by the RNase $\mathrm{Z}$ which does not contain any RNA components. RNase $\mathrm{Z}$ is responsible for the processing of tRNA precursors lacking an encoded CCA 3 'terminus [107]. In E. coli all tRNA transcripts are terminated on CCA and it has been suggested that RNase $\mathrm{Z}$ plays a role in RNA decay [108], while in Bacillus subtilis, the best studied of the Gram-positive bacteria, about two-thirds of tRNAs (59 out of 86) have the CCA motif encoded by their genes and the presence of RNase $\mathrm{Z}$ gene is essential [107]. In $S$. mutans UA159 the RNase Z is encoded by SMU1474 gene. As only 9 out of 65 identified tRNA molecules are terminated on CCA the RNase Z plays a very important role in that strain.

\subsection{Transfer-Messenger RNA-tmRNA}

Transfer-messenger RNA (ssr A) is a bacterial RNA molecule with dual tRNA-like and messenger RNA-like properties. The tmRNA forms a ribonucleoprotein complex (tmRNP) together with Small Protein B (SmpB), Elongation Factor Tu (EF-Tu), and ribosomal protein S1. In trans-translation, tmRNP binds to bacterial ribosomes which have arrested on mRNA due to the presence of rare codons, lack of specific charged tRNA rare or stop codon. The tmRNA is remarkably versatile: it recycles the stalled ribosome, adds a proteolysis-inducing tag to the unfinished polypeptide, and facilitates the degradation of the aberrant messenger RNA [109] [110] [111].

Both ssrA (1139684-1139337) and $\operatorname{smp} B$ (SMU1606) genes have been identified on $S$. mutans UA159 chromosome [2]. However tmRNA functions mostly as a quality control and "recycling" pathway in some bacteria it can modulate gene activity acting as asRNA and binding to the RBS sites [112] and is required for full virulence, either enhancing or repressing the expression of specific genes during infection [113]. As the oral environment undergoes constant changes in nutrient availability it is likely that tmRNA plays an important role in $\mathcal{S}$. mutans and other oral bacteria, however no experimental data are currently available. It has been shown that proteins tagged by $s s r A$ are degraded by ClpP protease complex in $S$. mutans (Tao and Biswas, unpublished). As $c l p P$ deletion induces a striking difference in gene expression [99] and affects many physiological traits [114] it is possible that tmRNA plays also a role in gene regulation.

\subsection{S/SsrS RNA}

The 6S RNA specifically associates with RNA polymerase holoenzyme containing the sigma70 specificity factor. This interaction represses expression mostly from $\sigma^{70}$-dependent (so called $\sigma^{70}$ extended promoters) but also some $\sigma^{32}$ promoters during stationary phase [115] [116]. B. subtilis and several closely related bacteria have two 6S RNAs that are differentially expressed suggesting they might act at different stages of growth [116] [117]. 
In $S$. mutans only one $s s r S$ gene has been identified (pos. 1927946-1928139). It is interesting that $s s r S$ gene overlaps (39 nt) in antisens orientation with $r p o B$ gene encoding RNA polymerase B subunit. Recently it has been shown that $E$. coli 6S RNA affects the amount of global stress alarmon ppGpp and it has been suggested that 6S RNA modulates transcription of several global regulators directly, including relA, to downregulate expression of key pathways in response to changing environmental conditions [118].

\subsection{Signal Recognition Particle RNA-SRP RNA}

SRP RNA is the RNA component of the signal recognition particle (SRP) ribonucleoprotein complex. SRP is universally conserved between many kingdoms and functions as a chaperone that directs the traffic of proteins within the cell and allows them to be secreted. The SRP RNA, together with one or more SRP proteins contributes to the binding and release of the signal peptide. SRP RNA is responsible for interaction and conformational changes of SRP and membrane receptor SM protein [119] [120].

In $S$. mutans SRP RNA have been identified in the SMU305-SMU307 (pos.293137-293237) and the SRP protein is encoded by SMU1060 (ffh) gene [2]. The two SM proteins, YidC1 and YidC2, are present and expressed in S. mutans. Deletion of YidC1 demonstrated no obvious phenotype. Elimination of YidC2 resulted in a stress-sensitive phenotype similar to SRP pathway mutants. In contrary to $E$. coli and B. subtilis mutations in $S$. mutans SRP RNA and/or ffh genes was not lethal but affected cells viability in stress conditions [121] [122]. That emphasizes the role of SRP mechanism in stress response processes and pointed out the differences between $S$. mutans and other model organisms.

\section{Future Prospects}

Posttranscriptional regulation of gene expression plays an important role in cell physiology of all living organisms. The most important role in that process is being held by small RNA molecules. Current technology allows identifying those small molecules; however functions of many sRNA still remain unknown. For some human pathogens like $S$. mutans which is naturally able to uptake any extracellular DNA an antisense therapy targeting sRNA might be a method of choice. That technique has been used effectively in prokaryotic systems, including S. mutans to inhibit gene expression [123]-[128]. Recently, more stable, chemically modified oligonucleotides (phosphorothioate oligodeoxyribonucleotides, PS-ODNs) have been used for gene silencing in S. mutans [129] [130]. Developing new strategies for delivery of those oligonucleotides and selecting the most appropriate targets might be one of the major goals to overcome the problem of increased multidrug-resistance in pathogenic microorganisms.

\section{Acknowledgements}

I want to thank Dr. Garth Ehrlich, FAAAS, Professor of Microbiology and Immunology, Professor of Otolaryngology-Head and Neck Surgery, Executive Di- 
rector for the Center for Advanced Microbial Processing (CAMP), Center for Genomic Sciences, Genomics Core Facility, Clinical and Translational Research Institute, Drexel College of Medicine for the financial support in publishing this paper.

\section{References}

[1] Lemos, J.A., et al. (2013) Streptococcus mutans: A New Gram-Positive Paradigm? Microbiology, 159, 436-445. https://doi.org/10.1099/mic.0.066134-0

[2] Ajdic, D. et al. (2002) Genome Sequence of Streptococcus mutans UA159, a Cariogenic Dental Pathogen. Proceedings of the National Academy of Sciences of the United States of America, 99, 14434-14439. https://doi.org/10.1073/pnas.172501299

[3] Lemos, J.A. and Burne, R.A. (2008) A Model of Efficiency: Stress Tolerance by Streptococcus mutans. Microbiology, 154, 3247-3255.

https://doi.org/10.1099/mic.0.2008/023770-0

[4] Banas, J.A. and Vickerman, M.M. (2003) Glucan-Binding Proteins of the Oral Streptococci. Critical Reviews in Oral Biology \& Medicine, 14, 89-99. https://doi.org/10.1177/154411130301400203

[5] Matsui, R. and Cvitkovitch, D. (2010) Acid Tolerance Mechanisms Utilized by Streptococcus mutans. Future Microbiology, 5, 403-417.

[6] Colby, S.M. and Russell, R.R.B. (1997) Sugar metabolism by Streptococci mutans. Journal of Applied Microbiology, 83, 80-88. https://doi.org/10.1046/j.1365-2672.83.s1.9.x

[7] Nishimura, J., et al. (2012) Biofilm Formation by Streptococcus mutans and Related Bacteria. Advances in Applied Microbiology, 2, 208-215.

[8] Kamiya, R.U., Taiete, T. and Gonçalves, R.B. (2011) Mutacins of Streptococcus mutans. Brazilian Journal of Microbiology, 42, 1248-1258. https://doi.org/10.1590/S1517-83822011000400001

[9] Bowen, W.H. and Koo, H. (2011) Biology of Streptococcus mutans-Derived Glucosyltransferases: Role in Extracellular Matrix Formation of Cariogenic Biofilms. Caries Research, 45, 69-86. https://doi.org/10.1159/000324598

[10] Merritt, J. and Qi, F. (2012) The Mutacins of Streptococcus mutans: Regulation and Ecology. Molecular Oral Microbiology, 27, 57-69. https://doi.org/10.1111/j.2041-1014.2011.00634.x

[11] Eckert, R., et al. (2006) Targeted Killing of Streptococcus mutans by a Pheromone-Guided "Smart" Antimicrobial Peptide. Antimicrobial Agents and Chemotherapy, 50, 3651-3657. https://doi.org/10.1128/AAC.00622-06

[12] Chen, F. and Wang, D. (2010) Novel Technologies for the Prevention and Treatment of Dental Caries: A Patent Survey. Expert Opinion on Therapeutic Patents, 20, 681-694. https://doi.org/10.1517/13543771003720491

[13] Burne, R.A., et al. (2009) Opportunities for Disrupting Cariogenic Biofilms. Advanced Dental Research, 21, 17-20. https://doi.org/10.1177/0895937409335593

[14] Hillman, J.D., et al. (2007) Modification of an Effect or Strain for Replacement Therapy of Dental Caries to Enable Clinical Safety Trials. Journal of Applied Microbiology, 102, 1209-1219. https://doi.org/10.1111/j.1365-2672.2007.03316.x

[15] Arraiano, C.M., et al. (2010) The Critical Role of RNA Processing and Degradation in the Control of Gene Expression. FEMS Microbiology Reviews, 34, 883-923. https://doi.org/10.1111/j.1574-6976.2010.00242.x 
[16] Winkler, W.C. and Breaker, R.R. (2005) Regulation of Bacterial Gene Expression by Riboswitches. Annual Review of Microbiology, 59, 487-517. https://doi.org/10.1146/annurev.micro.59.030804.121336

[17] Geissmann, T., Marzi, S. and Romby, P. (2009) The Role of mRNA Structure in Translational Control in Bacteria. RNA Biology, 6, 153-160. https://doi.org/10.4161/rna.6.2.8047

[18] Vasudevan, S. (2012) Posttranscriptional Up Regulation by Micro RNAs. Wiley Interdisciplinary Reviews. RNA, 3, 311-330. https://doi.org/10.1002/wrna.121

[19] Mager, W.H. (1988) Control of Ribosomal Protein Gene Expression. Biochimica et Biophysica Acta, 949, 1-15. https://doi.org/10.1016/0167-4781(88)90048-6

[20] Xue, X., et al. (2011) Lack of the Delta Subunit of RNA Polymerase Increases Virulence Related Traits of Streptococcus mutans. PLoS ONE, 6, Article ID: e20075. https://doi.org/10.1371/journal.pone.0020075

[21] Xue, X., et al. (2010) The Delta Subunit of RNA Polymerase, RpoE, Is a Global Modulator of Streptococcus mutans Environmental Adaptation. Journal of Bacteriology, 192, 5081-5092. https://doi.org/10.1128/JB.00653-10

[22] Smith, E.G. and Spatafora, G.A. (2012) Gene Regulation in S. mutans. Complex Control in a Complex Environment. Journal of Dental Research, 91, 133-141. https://doi.org/10.1177/0022034511415415

[23] Santangelo, T.J. and Artsimovitch, I. (2011) Termination and Antitermination: RNA Polymerase Runs a Stop Sign. Nature Reviews Microbiology, 9, 319-329. https://doi.org/10.1038/nrmicro2560

[24] Henkin, T.M. (2000) Transcription Termination Control in Bacteria. Current Opinion in Microbiology, 3, 149-153. https://doi.org/10.1016/S1369-5274(00)00067-9

[25] Yanofsky, C. (2000) Transcription Attenuation: Once Viewed as a Novel Regulatory Strategy. Journal of Bacteriology, 182, 1-8. https://doi.org/10.1128/JB.182.1.1-8.2000

[26] Babitzke, P., Baker, C.S. and Romeo, T. (2009) Regulation of Translation Initiation by RNA Binding Proteins. Annual Review of Microbiologym, 63, 27-44.

[27] Desnoyers, G., Bouchard, M.P. and Massé, E. (2013) New Insights into Small RNA-Dependent Translational Regulation in Prokaryotes. Trends in Genetics, 29, 92-98. https://doi.org/10.1016/j.tig.2012.10.004

[28] Lalaouna, D., et al. (2013) Regulatory RNAs and target mRNA Decay in Prokaryotes. Biochimica et Biophysica Acta, 1829, 742.

https://doi.org/10.1016/j.bbagrm.2013.02.013

[29] Wagner, E.G.H. and Simons, R.W. (1994) Antisense RNA Control in Bacteria, Phages, and Plasmids. Annual Review of Microbiology, 48, 713-742. https://doi.org/10.1146/annurev.mi.48.100194.003433

[30] Mahadevan, S. (1997) The BglG Group of Antiterminators: A Growing Family of Bacterial Regulators. Journal of Bioscience and Bioengineering, 22, 505-513. https://doi.org/10.1007/BF02703196

[31] Yang, Y., et al. (2002) Solution Structure of the LicT-RNA Antitermination Complex: CAT Clamping RAT. The EMBO Journal, 21, 1987-1997.

[32] Ajdić, D. and Pham, V.T.T. (2007) Global Transcriptional Analysis of Streptococcus mutans Sugar Transporters Using Microarrays. Journal of Bacteriology, 189, 5049-5059. https://doi.org/10.1128/JB.00338-07

[33] Old, L.A., Lowes, S. and Russell, R.R.B. (2006) Genomic Variation in Streptococcus mutans. Deletions Affecting the Multiple Pathways of $\beta$-Glucoside Metabolism. Oral Microbiology and Immunology, 21, 21-27. 
[34] Cote, C.K. and Honeyman, A.L. (2002) The Transcriptional Regulation of the Streptococcus mutans Bgl Regulon. Oral Microbiology and Immunology, 17, 1-8. https://doi.org/10.1046/j.0902-0055.2001.00087.x

[35] Cote, C.K. and Honeyman, A.L. (2003) The LicT Protein Acts as Both a Positive and a Negative Regulator of Loci within the Bgl Regulon of Streptococcus mutans. Microbiology, 149, 1333-1340.

[36] Cote, C.K. and Honeyman, A.L. (2006) Transcriptional Analysis of the Bglp Gene from Streptococcus mutans. BMC Microbiology, 6, 37.

https://doi.org/10.1186/1471-2180-6-37

[37] Burne, R.A., et al. (1999) Regulation of Expression of the Fructan Hydrolase Gene of Streptococcus mutans GS-5 by Induction and Carbon Catabolite Repression. Journal of Bacteriology, 181, 2863-2871.

[38] Wen, Z.T. and Burne, R.A. (2002) Analysis of Cis- and Trans-Acting Factors Involved in Regulation of the Streptococcus mutans Fructanase Gene (Frua). Journal of Bacteriology, 184, 126-33.

[39] Zeng, L. and Burne, R.A. (2009) Transcriptional Regulation of the Cellobiose Operon of Streptococcus mutans. Journal of Bacteriology, 191, 2153-2162.

[40] Henstra, S.A., et al. (1999) The Bacillus Stearothermophilus Mannitol Regulator, MtlR, of the Phosphotransferase system: A DNA-Binding Protein, Regulated by $\mathrm{Hpr}$ and IICBmtl-dependent Phosphorylation. The Journal of Biological Chemistry, 274, 4754-4763. https://doi.org/10.1074/jbc.274.8.4754

[41] Tan, K., et al. (2009) The Mannitol Operon Repressor MtlR Belongs to a New Class of Transcription Regulators in Bacteria. The Journal of Biological Chemistry, 284, 36670-36679. https://doi.org/10.1074/jbc.M109.062679

[42] Friedman, D.I., et al. (1989) Genetic Analysis of the N Transcription Antitermination System of Phage Lambda. Genome, 31, 491-496.

https://doi.org/10.1139/g89-096

[43] Arnvig, K.B., et al. (2008) Evolutionary Comparison of Ribosomal Operon Antitermination Function. Journal of Bacteriology, 190, 7251-7257. https://doi.org/10.1128/JB.00760-08

[44] Morgan, E.A. (1986) Antitermination Mechanisms in rRNA Operons of Escherichia coli. Journal of Bacteriology, 168, 1-5. https://doi.org/10.1128/jb.168.1.1-5.1986

[45] Berg, K.L., Squires, C. and Squires, C.L. (1989) Ribosomal RNA Operon Anti-Termination: Function of Leader and Spacer Region Box B-Box a Sequences and Their Conservation in Diverse Micro-Organisms. Journal of Molecular Biology, 209, 345-358.

[46] Doherty, G.P., Meredith, D.H. and Lewis, P.J. (2006) Subcellular Partitioning of Transcription Factors in Bacillus subtilis. Journal of Bacteriology, 188, 4101-4110. https://doi.org/10.1128/JB.01934-05

[47] Switzer, R.L., Turner, R.J. and Lu, Y. (1999) Regulation of the Bacillus subtilis Pyrimidine Biosynthetic Operon by Transcriptional Attenuation: Control of Gene Expression by an mRNA-Binding Protein. Prog. Progress in Nucleic Acid Research and Molecular Biology, 62, 329-67. https://doi.org/10.1016/S0079-6603(08)60512-7

[48] Lu, Y., Turner, R.J. and Switzer, R.L. (1996) Function of RNA Secondary Structures in Transcriptional Attenuation of the Bacillus subtilis Pyr Operon. Proceedings of the National Academy of Sciences of the United States of America, 93, 14462-14467.

[49] Bonner, E.R., et al. (2001) Molecular Recognition of Pyr mRNA by the Bacillus subtilis Attenuation Regulatory Protein PyrR. Nucleic Acids Research, 29, 4851-4865. https://doi.org/10.1093/nar/29.23.4851 
[50] Zengel, J.M. and Lindahl, L. (1994) Diverse Mechanisms for Regulating Ribosomal Protein Synthesis in Escherichia Coli. Progress in Nucleic Acid Research and Molecular Biology, 47, 331-370.

[51] Iben, J.R. and Draper, D.E. (2008) Specific Interactions of the L10(L12)4 Ribosomal Protein Complex with mRNA, rRNA, and L11. Biochemistry, 47, 2721-2731.

[52] Nomura, M. (1999) Regulation of Ribosome Biosynthesis in Escherichia Coli and Saccharomyces cerevisiae: Diversity and Common Principles. Journal of Bacteriology, 181, 6857-64.

[53] Henkin, T.M. (2008) Riboswitch RNAs: Using RNA to Sense Cellular Metabolism. Genes \& Development, 22, 3383-3390. https://doi.org/10.1101/gad.1747308

[54] Wakeman, C.A., Winkler, W.C. and Dann Iii, C.E. (2007) Structural Features of Metabolite-Sensing Riboswitches. Trends in Biochemical Sciences, 32, 415-424. https://doi.org/10.1016/j.tibs.2007.08.005

[55] Grundy, F.J. and Henkin, T.M. (2004) Regulation of Gene Expression by Effectors that Bind to RNA. Current Opinion in Microbiology, 7, 126-131. https://doi.org/10.1016/j.mib.2004.02.013

[56] Nudler, E. and Mironov, A.S. (2004) The Riboswitch Control of Bacterial Metabolism. Trends in Biochemical Sciences, 29, 11-17. https://doi.org/10.1016/j.tibs.2003.11.004

[57] Schyns, G., et al. (2005) Isolation and Characterization of New Thiamine-Deregulated Mutants of Bacillus subtilis. Journal of Bacteriology, 187, 8127-8136.

[58] Vogl, C., et al. (2007) Characterization of Riboflavin (Vitamin B2) Transport Proteins from Bacillus subtilis and Corynebacterium glutamicum. Journal of Bacteriology, 189, 7367-7375.

[59] Mandal, M., et al. (2004) A Glycine-Dependent Riboswitch that Uses Cooperative Binding to Control Gene Expression. Science, 306, 275-279.

[60] Barrick, J.E., et al. (2004) New RNA Motifs Suggest an Expanded Scope for Riboswitches in Bacterial Genetic Control. Proceedings of the National Academy of Sciences of the United States of America, 101, 6421-6426.

[61] Cromie, M.J., et al. (2006) An RNA Sensor for Intracellular Mg2+. Cell, 125, 71-84. https://doi.org/10.1016/j.cell.2006.01.043

[62] Fuchs, R.T., Grundy, F.J. and Henkin, T.M. (2006) The SMK Box Is a New SAM-Binding RNA for translational Regulation of SAM Synthetase. Nature Structural \& Molecular Biology, 13, 226-233. https://doi.org/10.1038/nsmb1059

[63] Jeremiah J., et al. (1993) The Structure of a Tetrahydrofolate-Sensing Riboswitch Reveals Two Ligand Binding Sites in a Single Aptamer. Structure, 19, 1413-1423.

[64] Ames, T.D., et al. (2010) A Eubacterial Riboswitch Class that Senses the Coenzyme Tetrahydrofolate. Nature Chemical Biology, 17, 681-685. https://doi.org/10.1016/j.chembiol.2010.05.020

[65] Baker, J.L., et al. (2012) Widespread Genetic Switches and Toxicity Resistance Proteins for Fluoride. Science, 335, 233-235. https://doi.org/10.1126/science.1215063

[66] Marquis, R.E. (1995) Antimicrobial Actions of Fluoride for oral Bacteria. Canadian Journal of Microbiology, 41, 955-964.

[67] Green, N.J., Grundy, F.J. and Henkin, T.M. (2010) The T Box Mechanism: tRNA as a Regulatory Molecule. FEBS Letters, 584, 318-324. https://doi.org/10.1016/j.febslet.2009.11.056

[68] Henkin, T.M. (1994) Micro Review tRNA-dircted Transcription Antitermination. 
Molecular Microbiology, 13, 381-387.

https://doi.org/10.1111/j.1365-2958.1994.tb00432.x

[69] Gutiérrez-Preciado, A., et al. (2009) Biochemical Features and Functional Implications of the RNA-based T-Box regulatory mechanism. Microbiology and Molecular Biology Reviews, 73, 36-61. https://doi.org/10.1128/MMBR.00026-08

[70] Gottesman, S. and Storz, G. (2011) Bacterial small RNA regulators: versatile roles and rapidly evolving variations. Cold Spring Harbor Perspectives in Biology, 3.

[71] Wagner, E.G.H., Altuvia, S. and Romby, P. (2002) 12 Antisense RNAs in Bacteria and Their Genetic Elements. In: Advances in Genetics, Jay, C.D. and Wu, C.T., Ed., Academic Press, 361-398. https://doi.org/10.1016/S0065-2660(02)46013-0

[72] Fukuda, Y., Nakayama, Y. andTomita, M. (2003) On Dynamics of Overlapping Genes in Bacterial Genomes. Gene, 323, 181-187.

https://doi.org/10.1016/j.gene.2003.09.021

[73] Yamaguchi, Y., Park, J.H. and Inouye, M. (2011) Toxin-Antitoxin Systems in Bacteria and Archaea. Annual Review of Genetics, 45, 61-79.

https://doi.org/10.1146/annurev-genet-110410-132412

[74] Gerdes, K. and Maisonneuve, E. (2012) Bacterial Persistence and Toxin-Antitoxin Loci. Annual Review of Microbiology, 66, 103-123. https://doi.org/10.1146/annurev-micro-092611-150159

[75] Blower, T.R., et al. (2012) Identification and Classification of Bacterial Type III Toxin-Antitoxin Systems Encoded in Chromosomal and Plasmid Genomes. Nucleic Acids Research.

[76] Brantl, S. (2012) Bacterial Type I Toxin-Antitoxin Systems. RNA Biology, 9, 1488-1490. https://doi.org/10.4161/rna.23045

[77] Leplae, R., et al. (2011) Diversity of Bacterial Type II Toxin-Antitoxin Systems: A Comprehensive Search and Functional Analysis of Novel Families. Nucleic Acids Research, 39, 5513-5525. https://doi.org/10.1093/nar/gkr131

[78] Masuda, H., et al. (2012) YeeU Enhances the Bundling of Cytoskeletal Polymers of MreB and FtsZ, Antagonizing the CbtA (YeeV) Toxicity in Escherichia Coli. Molecular Microbiology, 84, 979-989. https://doi.org/10.1111/j.1365-2958.2012.08068.x

[79] Wang, X., et al. (2012) A New type V Toxin-Antitoxin System Where mRNA for Toxin GhoT Is Cleaved by Antitoxin GhoS. Chemistry \& Biology, 8, 855-861. https://doi.org/10.1038/nchembio.1062

[80] Fineran, P.C., et al. (2009) The Phage Abortive Infection System, Tox IN, Functions as a Protein-RNA Toxin-Antitoxin Pair. Proceedings of the National Academy of Sciences of the United States of America, 106, 894-899. https://doi.org/10.1073/pnas.0808832106

[81] Fozo, E.M., Hemm, M.R. and Storz, G. (2008) Small Toxic Proteins and the Antisense RNAs that Repress Them. Microbiology and Molecular Biology Reviews, 72, 579-589. https://doi.org/10.1128/MMBR.00025-08

[82] Rheinberg, A., et al. (2013) Cryptic Streptococcus mutans 5.6-kb Plasmids Encode a Toxin-Antitoxin System for Plasmid Stabilization. Journal of Oral Microbiology, 5.

[83] Koyanagi, S. and Levesque, C.M. (2013) Characterization of a Streptococcus mutans Intergenic Region Containing a Small Toxic Peptide and Its Cis-Encoded Antisense Small RNA Antitoxin. PLoS ONE, 8, Article ID: e54291. https://doi.org/10.1371/journal.pone.0054291

[84] Gottesman, S. (2005) Micros for Microbes: Non-Coding Regulatory RNAs in Bacteria. Trends in Genetics, 21, 399-404. https://doi.org/10.1016/j.tig.2005.05.008 
[85] Romby, P., Vandenesch, F. and Wagner, E.G.H. (2006) The Role of RNAs in the Regulation of Virulence-Gene Expression. Current Opinion in Microbiology, 9 229-236.

[86] Papenfort, K. and Vogel, J. (2010) Regulatory RNA in Bacterial Pathogens. Cell Host \& Microbe, 8, 116-127. https://doi.org/10.1016/j.chom.2010.06.008

[87] Panja, S. and Woodson, S.A. (2012) Hfq Proximity and Orientation Controls RNA Annealing. Nucleic Acids Research.

[88] Møller, T., et al. (2002) Hfq: A Bacterial Sm-Like Protein that Mediates RNA-RNA Interaction. Molecular Cell, 9, 23-30.

[89] Mangold, M., et al. (2004) Synthesis of Group a Streptococcal Virulence Factors Is Controlled by a Regulatory RNA Molecule. Molecular Microbiology, 53, 1515-1527. https://doi.org/10.1111/j.1365-2958.2004.04222.x

[90] Ramirez-Peña, E., et al. (2010) The Group A Streptococcus Small Regulatory RNA FasX Enhances Streptokinase Activity by Increasing the Stability of the Ska mRNA Transcript. Molecular Microbiology, 78, 1332-1347.

[91] Romby, P. and Charpentier, E. (2010) An Overview of RNAs with Regulatory Functions in Gram-Positive Bacteria. Cellular and Molecular Life Sciences, 67, 217-237. https://doi.org/10.1007/s00018-009-0162-8

[92] Perez, N., et al. (2009) A Genome-Wide Analysis of Small Regulatory RNAs in the Human Pathogen Group a Streptococcus. PLoS ONE, 4, Article ID: e7668. https://doi.org/10.1371/journal.pone.0007668

[93] Lemme, A., et al. (2011) Subpopulation-Specific Transcriptome Analysis of Competence-Stimulating-Peptide-Induced Streptococcus mutans. Journal of Bacteriology, 193, 1863-1877.

[94] Marraffini, L.A. and Sontheimer, E.J. (2010) CRISPR Interference: RNA-Directed Adaptive Immunity in Bacteria and Archaea. Nature Reviews. Genetics, 11, 181-190.

[95] Haft, D.H., et al. (2005) A Guild of 45 CRISPR-Associated (Cas) Protein Families and Multiple CRISPR/Cas Subtypes Exist in Prokaryotic Genomes. Plos Computational Biology, 1, e60. https://doi.org/10.1371/journal.pcbi.0010060

[96] Grissa, I., Vergnaud, G. and Pourcel, C. (2007) The CRISPRdb Database and Tools to Display CRISPRs and to Generate Dictionaries of Spacers and Repeats. BMC Bioinformatics, 8, 172.

[97] Deltcheva, E., et al. (2011) CRISPR RNA Maturation by Trans-Encoded Small RNA and Host Factor RNase III. Nature, 471, 602-607.

https://doi.org/10.1038/nature09886

[98] Van der Ploeg, J.R. (2009) Analysis of CRISPR in Streptococcus mutans Suggests Frequent Occurrence of Acquired Immunity against Infection by M102-Like Bacteriophages. Microbiology, 155, 1966-1976. https://doi.org/10.1099/mic.0.027508-0

[99] Chattoraj, P., et al. (2010) ClpP of Streptococcus mutans Differentially Regulates Expression of Genomic Islands, Mutacin Production and Antibiotic Tolerance. Journal of Bacteriology, 192, 1312-1323. https://doi.org/10.1128/JB.01350-09

[100] Halfmann, A., et al. (2007) Identification of the Genes Directly Controlled by the Response Regulator CiaR in Streptococcus Pneumoniae: Five Out of 15 Promoters Drive Expression of Small Non-Coding RNAs. Molecular Microbiology, 66, 110-126. https://doi.org/10.1111/j.1365-2958.2007.05900.x

[101] Göpel, Y. and Görke, B. (2012) Rewiring Two-Component Signal Transduction with Small RNAs. Current Opinion in Microbiology, 15, 132-139.

https://doi.org/10.1016/j.mib.2011.12.001 
[102] Bushati, N. and Cohen, S.M. (2007) microRNA functions. Annual Review of Cell and Developmental Biology, 23, 175-205.

https://doi.org/10.1146/annurev.cellbio.23.090506.123406

[103] He, L. and Hannon, G.J. (2004) MicroRNAs: Small RNAs with a Big Role in Gene Regulation. Nature Reviews. Genetics, 5, 522-531.

[104] Filipowicz, W., Bhattacharyya, S.N. and Sonenberg, N. (2008) Mechanisms of Post-Transcriptional Regulation by microRNAs: Are the Answers in Sight? Nature Reviews. Genetics, 9, 102-114.

[105] Lee, H.J. and Hong, S.H. (2012) Analysis of microRNA-Size, Small RNAs in Streptococcus mutans by deep Sequencing. FEMS Microbiology Letters, 326, 131-136. https://doi.org/10.1111/j.1574-6968.2011.02441.x

[106] Guerrier-Takada, C., et al. (1983) The RNA Moiety of Ribonuclease P Is the Catalytic Subunit of the Enzyme. Cell, 35, 849-857. https://doi.org/10.1016/0092-8674(83)90117-4

[107] Pellegrini, O., et al. (2003) Endonucleolytic Processing of CCA-less tRNA Precursors by RNase Z in Bacillus subtilis. EMBO Journal, 22, 4534-4543.

[108] Perwez, T. and Kushner, S.R. (2006) RNase Z in Escherichia Coli Plays a Significant Role in mRNA Decay. Molecular Microbiology, 60, 723-737.

[109] Keiler, K.C. (2008) Biology of Trans-Translation. Annual Review of Microbiology, 62, 133-151.

[110] Deana, A. and Belasco, J.G. (2005) Lost in Translation: the Influence of Ribosomes on Bacterial mRNA Decay. Genes \& Development, 19, 2526-2533. https://doi.org/10.1101/gad.1348805

[111] Gillet, R. and Felden, B. (2001) Emerging Views on tmRNA-Mediated Protein Tagging and Ribosome Rescue. Molecular Microbiology, 42, 879-885. https://doi.org/10.1046/j.1365-2958.2001.02701.x

[112] Liu, Y., et al. (2010) SSRA (tmRNA) Acts as an Antisense RNA to Regulate Staphylococcus Aureus Pigment Synthesis by Base Pairing with crtMN mRNA. FEBS Letter, 584, 4325-4329. https://doi.org/10.1016/j.febslet.2010.09.024

[113] Julio, S.M., Heithoff, D.M. and Mahan, M.J. (2000) SSRA (tmRNA) Plays a Role in Salmonella Enterica Serovar Typhimurium Pathogenesis. Journal of Bacteriology, 182, 1558-1563. https://doi.org/10.1128/JB.182.6.1558-1563.2000

[114] Lemos, J.A.C. and Burne, R.A. (2002) Regulation and Physiological Significance of $\mathrm{ClpC}$ and ClpP in Streptococcus mutans. Journal of Bacteriology, 184, 6357-6366. https://doi.org/10.1128/JB.184.22.6357-6366.2002

[115] Wassarman, K.M. and Storz, G. (2000) 6S RNA Regulates E. coli RNA Polymerase Activity. Cell, 101, 613-623. https://doi.org/10.1016/S0092-8674(00)80873-9

[116] Wassarman, K.M. (2007) 6S RNA: A Small RNA Regulator of Transcription. Current Opinion in Microbiology, 10, 164-168.

https://doi.org/10.1016/j.mib.2007.03.008

[117] Cavanagh, A.T., Sperger, J.M. and Wassarman, K.M. (2012) Regulation of 6S RNA by pRNA Synthesis Is Required for Efficient Recovery from Stationary Phase in E. Coli and B. subtilis. Nucleic Acids Research, 40, 2234-2246. https://doi.org/10.1093/nar/gkr1003

[118] Cavanagh, A.T., Chandrangsu, P. and Wassarman, K.M. (2010) 6S RNA Regulation of relA alters ppGpp Levels in Early Stationary Phase. Microbiology, 156, 3791-3800. https://doi.org/10.1099/mic.0.043992-0

[119] Neher, S.B., et al. (2008) SRP RNA Controls a Conformational Switch Regulating 
the SRP-SRP Receptor Interaction. Nature Structural \& Molecular Biology, 15, 916-923. https://doi.org/10.1038/nsmb.1467

[120] Shen, K., et al. (2012) Activated GTPase Movement on an RNA Scaffold Drives co-Translational Protein Targeting. Nature, 492, 271-275. https://doi.org/10.1038/nature11726

[121] Hasona, A., et al. (2005) Streptococcal Viability and Diminished Stress Tolerance in Mutants Lacking the Signal Recognition Particle Pathway or YidC2. Proceedings of the National Academy of Sciences of the United States of America, 102, 17466-17471. https://doi.org/10.1073/pnas.0508778102

[122] Funes, S., et al. (2009) Independent Gene Duplications of the YidC/Oxa/Alb3 Family Enabled a Specialized Cotranslational Function. Proceedings of the National Academy of Sciences of the United States of America, 106, 6656-6661. https://doi.org/10.1073/pnas.0809951106

[123] Wang, B. and Kuramitsu, H.K. (2005) Inducible Antisense RNA Expression in the Characterization of Gene Functions in Streptococcus mutans. Infection and Immunity, 73, 3568-3576. https://doi.org/10.1128/IAI.73.6.3568-3576.2005

[124] Man, S., et al. (2011) Artificial Trans-Encoded Small Non-Coding RNAs Specifically Silence the Selected Gene Expression in Bacteria. Nucleic Acids Research, 39, e50.

[125] Ji, Y., et al. (2001) Identification of Critical Staphylococcal Genes Using Conditional Phenotypes Generated by Antisense RNA. Science, 293, 2266-2269. https://doi.org/10.1126/science.1063566

[126] Gillaspie, D., et al. (2009) Plasmid-Based System for High-Level Gene Expression and Antisense Gene Knockdown in Bartonella Henselae. Applied and Environmental Microbiology, 75, 5434-5436. https://doi.org/10.1128/AEM.00949-09

[127] Kedar, G.C., et al. (2007) Evaluation of the metS and murB Loci for Antibiotic Discovery Using Targeted Antisense RNA Expression Analysis in Bacillus Anthracis. Antimicrobial Agents and Chemotherapy, 51, 1708-1718. https://doi.org/10.1128/AAC.01180-06

[128] Guo, Q.Y., et al. (2006) Treatment of Streptococcus mutans with Antisense Oligodeoxyribonucleotides to gtfb mRNA Inhibits GtfB Expression and Function. FEMS Microbiology Letters, 264, 8-14. https://doi.org/10.1111/j.1574-6968.2006.00378.x

[129] Dufour, M., McLeod, F.S.A. and Simmonds, R.S. (2011) A Facilitates the Entry of Antisense Constructs into Streptococcus mutans. FEMS Microbiology Letters, 317, 93-99. https://doi.org/10.1111/j.1574-6968.2011.02220.x

[130] McLeod, F. (2012) Entry of Antisense Oligonucleotides into Streptococcus mutans. University of Otago, Dunedin. 


\section{Abbreviations}

TCSTS-two component signal transduction systems

asRNA-antisense RNA

sRNA—small RNA

miRNA - micro RNA

RAT-ribonucleic antiterminator

CSP-competence-stimulating peptide

RNAi-interference RNA

TIR-translation initiation region

TA - toxin /antitoxin system

PTS-phosphotransferase systems 